\title{
Moderate mitral regurgitation in aortic root replacement surgery: Comparing mitral repair with no mitral repair
}

\author{
Fenton H. McCarthy, MD, Nimesh D. Desai, MD, PhD, Zachary Fox, BA, Justin George, BA, \\ Patrick Moeller, BS, Prashanth Vallabhajosyula, MD, Wilson Y. Szeto, MD, and Joseph E. Bavaria, MD
}

\begin{abstract}
Objectives: Patients often present for aortic root replacement surgery with concomitant mitral valve pathology. Moderate mitral regurgitation is the point of clinical equipoise where the benefits of intervention compared with observation are currently unknown. This study compares outcomes in patients undergoing aortic root replacement surgery who did or did not receive a mitral valve repair for their preoperative moderate mitral regurgitation.

Methods: A total of 1316 patients who underwent aortic root replacement surgery between 2000 and 2011 were evaluated, with 104 patients meeting the inclusion criteria by presenting with moderate preoperative mitral regurgitation. A total of 73 patients $(70 \%)$ received no mitral intervention, and 31 patients $(30 \%)$ received a mitral repair. Patients underwent preoperative, postoperative, and greater than 6-month follow-up echocardiograms. Average clinical follow-up was 6.5 years.
\end{abstract}

Results: The mitral repair group had increased preoperative New York Heart Association III/IV and heart failure, longer crossclamp times, and more postoperative renal failure $(P=.0003, P=.04, P<.0001$, and $P=.03$, respectively). The improvement in mitral regurgitation was greater for the mitral repair group $(-2.1 \pm 0.3$ vs $-1.1 \pm 0.8, P<.0001)$, and mitral regurgitation remained significantly lower on follow-up at 6 months or more $(0.6 \pm 0.40 .9 \pm 0.2, P=.002)$. A significantly greater percentage of patients undergoing mitral repair compared with patients with no repair had improvement of at least 1 grade in mitral regurgitation postoperatively $(100 \%$ vs $70 \%, P=.001)$ and on follow-up at 6 months or more $(90 \%$ vs $61 \%, P=.006)$. There was no difference in long-term survival, freedom from $2+$ or greater mitral regurgitation, or mitral reinterventions.

Conclusions: Mitral repair along with aortic root replacement has acceptable operative risk. Aortic root replacement surgery alone improved mitral regurgitation, but the addition of mitral repair further reduced mitral regurgitation, suggesting that repairing moderate mitral regurgitation should generally be considered along with aortic root replacement. (J Thorac Cardiovasc Surg 2014;147:938-41)

Patients frequently present with both aortic and mitral valve pathology. In published series of aortic valve replacement (AVR), as many as $61 \%$ to $90 \%$ of patients have some degree of mitral regurgitation (MR). ${ }^{1}$ Patients undergoing aortic root replacements also frequently present with MR because many of the degenerative diseases of the aortic root also affect the mitral valve. ${ }^{2}$ Consensus exists that severe MR requires intervention at the time of aortic intervention, but there is ongoing debate regarding the benefit of intervening on less severe MR. Recent literature also suggests that when mitral intervention is undertaken

From the Department of Cardiovascular Surgery, Hospital of the University of Pennsylvania, Philadelphia, Pa.

Disclosures: Authors have nothing to disclose with regard to commercial support.

Presented at The 2013 American Association for Thoracic Surgery Mitral Conclave, New York, New York, May 2, 2013.

Received for publication April 28, 2013; revisions received July 13, 2013; accepted for publication July 22, 2013; available ahead of print Sept 16, 2013.

Address for reprints: Fenton H. McCarthy, MD, Department of Surgical Education,

4 Maloney, Hospital of the University of Pennsylvania, 3400 Spruce St,

Philadelphia, PA 19104 (E-mail: Fenton.McCarthy@uphs.upenn.edu).

0022-5223/\$36.00

Copyright (c) 2014 by The American Association for Thoracic Surgery

http://dx.doi.org/10.1016/j.jtcvs.2013.07.056 for MR, mitral valve repairs are likely more beneficial than mitral valve replacements. ${ }^{3}$ Clinical equipoise still exists whether the conservative approach of observation or the interventional approach of mitral repair is best for patients with moderate MR undergoing aortic valve intervention. This study compares echocardiographic and clinical outcomes in patients undergoing aortic root replacement surgery who did or did not receive a mitral valve repair for their preoperative moderate MR.

\section{PATIENTS AND METHODS}

The institutional review board at the University of Pennsylvania approved the study and waived the need for patient consent. A total of 1316 patients who underwent aortic root replacement surgery between January 2000 and December 2011 were evaluated. Exclusion criteria included mitral stenosis, endocarditis, aortic dissection, and mitral replacement. A total of 104 patients met the inclusion criteria and presented preoperatively with moderate MR, defined as greater than mild (1+) and less than severe $(4+)$. A total of 73 patients $(70 \%)$ received no mitral intervention, and 31 patients (30\%) received a mitral repair.

All patients underwent preoperative and postoperative echocardiograms. Preoperative transthoracic echocardiograms (TTEs) were preferentially selected for all echocardiograms to provide consistent loading conditions and comparable parameters without the confounding effect of general anesthesia on the severity of MR. Transesophageal 


\section{Abbreviations and Acronyms}

AVR $=$ aortic valve replacement

$\mathrm{CABG}=$ coronary artery bypass grafting

$\mathrm{MR}=$ mitral regurgitation

TTE $=$ transthoracic echocardiogram

echocardiograms were used when TTEs were unavailable. Preoperative TTEs had to be performed within 6 months of the date of surgery. Postoperative TTEs had to occur before the patient left the hospital. Greater than 6-month echocardiograms were obtained on $62 \%(n=65)$ of patients with an average echocardiographic follow-up time of 3.3 years. If more than 1 follow-up echocardiogram was available, the most recent examination was used to show the greatest degree of ventricular remodeling, hemodynamic changes, and stability of mitral repairs. Data points were extrapolated from echocardiogram reports using the MR grades of mild $(1+)$, mild-moderate $(1.5+)$, moderate $(2+)$, moderatesevere $(3+)$, and severe $(4+)$. Median clinical follow-up was 6.5 years.

Statistical analysis was carried out using SPSS 19 (SPSS Inc, Chicago Ill). Categoric variables were compared using chi-square tests with the Fisher exact test. Continuous variables were analyzed using the Student $t$ test. For comparison of changes in ordinal categories, nonparametric Mann-Whitney tests were conducted. Long-term mortality and freedom from late events were computed using Kaplan-Meier estimators and log-rank tests.

\section{RESULTS}

The mitral repair group had increased incidence of preoperative New York Heart Association III/IV (63.6\% vs $31.5 \%, P<.001)$ and heart failure $(78.7 \%$ vs $64.3 \%$, $P=.04)$. There were no other significant differences in patient characteristics, preoperative comorbid conditions, or previous cardiothoracic interventions. Operative characteristics and perioperative outcomes are detailed in Table 1. The repair group had a higher percentage of mixed aortic insufficiency/stenosis than the no repair group $(36.3 \%$ vs $19.1 \%, P=.047)$. The presenting cause of mitral diseases was different for both groups, with fewer functional $(45 \%$ vs $75 \%, P=.007)$ and increased number of myxomatous $(32 \%$ vs $10 \%, P=.01)$ and rheumatic $(10 \%$ vs $0 \%$, $P=.02)$ mitral valves in the repair group. Crossclamp time was increased in the repair group $(244.8 \pm 42$ vs $179.6 \pm 50.6, P<.0001$ ), as was postoperative renal failure $(9 \%$ vs $1.3 \%, P=.03)$ despite no difference in preoperative renal failure ( $3 \%$ vs $8.2 \%, P=.43$ ). There were 3 operative deaths in the no repair group and zero operative deaths in the repair group, but this difference was not statistically significant.

The mean improvement in MR was greater in the mitral repair group compared with the no repair group postoperatively $(-2.1 \pm 0.3$ vs $-1.1 \pm 0.8, P<.0001)$ and at more than 6-month follow-up $(-1.8 \pm 0.6$ vs $-1.3 \pm 0.5$, $P<.0001)$. Mean MR remained significantly lower on more than 6-month follow-up $(0.6 \pm 0.40 .9 \pm 0.2$, $P=.002)$. A significantly greater percentage of those receiving mitral repair compared with no repair had improvement of at least 1 grade in MR postoperatively $(100 \%$ vs $70 \%, P=.001)$ and at more than 6 -month follow-up (90\% vs $61 \%, P=.006)$ (Figure 1). There was no difference in MR improvement in either group depending on the cause of the presenting MR, the type of repair, or concomitant coronary artery bypass grafting (CABG).

The long-term clinical outcomes of freedom from $2+$ or greater MR were $100 \%, 98.5 \% \pm 1.5 \%, 96.5 \% \pm 2.5 \%$, and $96.5 \% \pm 2.5 \%$ in the no repair group at $1,3,5$, and 8 years, respectively, and $93.2 \% \pm 4.7 \%$ for all time points in the repair group $(P=.32)$. Freedom from mitral interventions was $100 \%, 100 \%, 97.9 \% \pm 2.1 \%$, and $97.9 \% \pm 2.1 \%$ at $1,3,5$, and 8 years, respectively, in the no repair group and $96.4 \% \pm 3.4 \%$ for all time points in the repair group $(P=.49)$. Survival was $94.5 \% \pm 3 \%$, $90.2 \% \pm 4 \%, 83.2 \% \pm 4.7 \%$, and $81.2 \% \pm 5 \%$ at 1,3 , 5 , and 8 years, respectively, in the no repair group and $93.4 \% \pm 4.5 \%, 90.1 \% \pm 5.4 \%, 90.1 \% \pm 5.4 \%$, and $67.3 \% \pm 16.3 \%$ at $1,3,5$, and 8 years, respectively, in the repair group $(P=.93)$. There was a trend toward worse survival in the mitral repair group, although this difference is not statistically significant. There was 1 repair failure at 9 months leading to a mitral valve replacement. Two patients receiving no repair underwent mitral repair at 1 and 4.5 years after root replacement.

\section{DISCUSSION}

Patients frequently present with both aortic and mitral valve pathology. Management of double-valve disease remains clinically and technically challenging. The presence of MR affects operative planning for both mitral and aortic valves, because MR has been associated with decreased survival and increased mortality risk in AVR and aortic root replacement. ${ }^{4}$ Some hesitancy exists to perform concomitant mitral valve interventions, because some MR has been shown to improve with AVR alone, and simultaneous aortic and mitral valve procedures have been associated with a 2-fold increase in mortality over isolated AVR. ${ }^{5}$ On the other hand, unaddressed MR after AVR also has significant consequences and has been associated with increased long-term mortality. ${ }^{6}$

Consensus exists that severe MR requires intervention, but there is ongoing debate regarding less severe MR. Trace or minimal MR is mostly observed with the expectation that MR improves with aortic valve intervention alone. ${ }^{7}$ Clinical equipoise exists on the best approach to the patient with moderate MR undergoing aortic intervention. A number of series have evaluated moderate MR for patients undergoing AVR, but the issue of comparing intervention with observation has yet to be explored in patients undergoing aortic root replacement. Considering the increased operative complexity of aortic root replacement and the potential for aortic root replacements to affect the aortomitral continuity, the decision to perform concomitant 
TABLE 1. Operative characteristics and outcomes

\begin{tabular}{|c|c|c|c|}
\hline $\begin{array}{l}\text { Primary aortic } \\
\text { indication }\end{array}$ & $\begin{array}{l}\text { Mitral repair } \\
\quad(\mathbf{n}=\mathbf{3 1})\end{array}$ & $\begin{array}{c}\text { No mitral } \\
\text { repair }(n=73)\end{array}$ & $\begin{array}{c}P \\
\text { value }\end{array}$ \\
\hline Aneurysm & $60.6 \%(20)$ & $67.1 \%(49)$ & .83 \\
\hline AI & $45.4 \%(15)$ & $57.5 \%(42)$ & .39 \\
\hline AS & $12.1 \%(4)$ & $5.4 \%(4)$ & .23 \\
\hline Mixed AI/AS & $36.3 \%(12)$ & $19.1 \%(14)$ & .047 \\
\hline Redo root & $18.1 \%(6)$ & $13.6 \%(10)$ & .55 \\
\hline \multicolumn{4}{|l|}{ MR cause } \\
\hline Functional & $14(45 \%)$ & $54(73 \%)$ & .007 \\
\hline Myxomatous & $10(32 \%)$ & $7(10 \%)$ & .01 \\
\hline Ischemic & $4(13 \%)$ & $12(16 \%)$ & .77 \\
\hline Rheumatic/other & $3(10 \%)$ & $0(0 \%)$ & .02 \\
\hline \multicolumn{4}{|l|}{ Operation status } \\
\hline Elective & $72.7 \%(24)$ & $58.9 \%(43)$ & .13 \\
\hline Urgent & $15.1 \%(5)$ & $34.2 \%(25)$ & .11 \\
\hline Emergency & $6 \%(2)$ & $2.7 \%(2)$ & .59 \\
\hline \multicolumn{4}{|l|}{ Concomitant procedure } \\
\hline CABG & $15.1 \%(5)$ & $16.4 \%(12)$ & 1.00 \\
\hline Hemi/total arch & $45.4 \%(15)$ & $46.5 \%(34)$ & 1.00 \\
\hline Tricuspid & $3 \%(1)$ & $6.8 \%(5)$ & .66 \\
\hline Maze & $3 \%(1)$ & $4.1 \%(3)$ & 1.00 \\
\hline \multicolumn{4}{|l|}{ Outcome } \\
\hline Crossclamp time (min) & $244.8 \pm 42$ & $179.6 \pm 50.6$ & $<.0001$ \\
\hline Reoperative bleeding & $6 \%(2)$ & $4.1 \%(3)$ & .65 \\
\hline $\begin{array}{l}\text { Perioperative myocardial } \\
\text { infarction }\end{array}$ & $0 \%(0)$ & $1.3 \%(1)$ & 1.00 \\
\hline Deep sternotomy infection & $3 \%(1)$ & $0 \%(0)$ & .31 \\
\hline Infection, septicemia & $3 \%(1)$ & $1.3 \%(1)$ & .23 \\
\hline Stroke permanent & $0 \%(0)$ & $1.3 \%(1)$ & 1.00 \\
\hline Stroke transient & $0 \%(0)$ & $1.3 \%(1)$ & 1.00 \\
\hline Vent prolonged & $30.3 \%(10)$ & $20.5 \%(15)$ & .22 \\
\hline Renal failure & $9 \%(3)$ & $1.3 \%(1)$ & .03 \\
\hline Dialysis & $3 \%(1)$ & $1.3 \%(1)$ & .53 \\
\hline Anticoagulant event & $0 \%(0)$ & $0 \%(0)$ & 1.00 \\
\hline Tamponade & $0 \%(0)$ & $1.3 \%(1)$ & 1.00 \\
\hline Gastrointestinal event & $6 \%(2)$ & $2.7 \%(2)$ & .59 \\
\hline Atrial fibrillation & $12.1 \%(4)$ & $19.1 \%(14)$ & .79 \\
\hline Heart block & $15.1 \%(5)$ & $10.9 \%(8)$ & .54 \\
\hline Pneumonia & $9 \%(3)$ & $2.7 \%(2)$ & .17 \\
\hline Mortality & $0 \%(0)$ & $4.1 \%(3)$ & .55 \\
\hline
\end{tabular}

$A I$, Aortic insufficiency; $A S$, aortic stenosis; $C A B G$, coronary artery bypass grafting; $M R$, mitral regurgitation.

mitral repair for moderate MR at the time of aortic root replacement remains a challenging clinical question.

This study demonstrates good short- and long-term outcomes for aortic root replacements in patients with moderate MR, which is a group previously shown to be at higher surgical risk for both aortic root replacement and AVR. ${ }^{2,7,8}$ Operative mortality was $2.8 \%$ for both groups with zero operative deaths in the mitral repair group. Of the 31 patients who underwent a repair, at more than 6 months follow-up 1 patient had worse MR, 1 patient had return of moderate MR, and the remainder had mild MR or less. There was a trend toward worse long-term survival in the mitral repair group. This difference was not statistically significant, and it is difficult to interpret this trend in the setting of an increased proportion of New York Heart Association III/IV and heart failure in the mitral repair group.

The group of patients who did not undergo mitral repair also demonstrated improvement in MR postoperatively and at more than 6 months. These results are consistent with other studies that have shown significant MR improvement with isolated AVR or AVR and CABG. In the no mitral repair group, $80 \%$ of patients demonstrated postoperative improvement in MR and $60 \%$ had $0+$ or $1+$ MR at more than 6 months follow-up. The results show more improvement in MR than some other AVR studies. ${ }^{1,9}$ One explanation for this could be the additional aortomitral continuity support provided by aortic root replacement. The observed group also did not demonstrate any significant short- or long-term clinical morbidity or mortality as a result of a conservative approach to managing their moderate MR.

With regard to the cause of the MR and concomitant procedures performed, mitral repair was attempted in $59 \%(n=10)$ of patients with myxomatous disease, and all myxomatous repairs had mild or less MR postoperatively and at more than 6 months except 1 patient with $2+$ MR detected at 2 years. Of the patients presenting with functional MR, 79\% were observed with no mitral repair, but of those patients undergoing repair $(n=14)$, similar improvement in MR as the repaired myxomatous valves was demonstrated. This study also included ischemic MR and patients who underwent concomitant CABG. This is potentially a confounding factor, because ischemic MR has been shown to improve with CABG. ${ }^{9}$ In this study, there was no difference in the number of ischemic and concomitant $\mathrm{CABG}$ cases in the repair and no repair groups or any increased improvement in MR when compared with nonischemic cases.

A number of studies have attempted to define which group of patients with MR will improve with just aortic or aortic and CABG interventions. ${ }^{1,8,10}$ These studies have not arrived at a reproducible set of similar preoperative factors that can be used to predict whose MR will improve without mitral valve intervention and indeed often have contradictory results. Failing to intervene on MR during aortic root replacement has the potential to subject the patient to the increased risk of ongoing MR and possible redo sternotomy if further mitral intervention is required. The surgeon also faces the increased technical difficulty of a mitral intervention with an aortic root replacement already present.

In this study, both repair and observation cohorts had good clinical outcomes, so no absolute indication for mitral valve repair or observation of moderate MR in aortic root replacement can be reasonably concluded on the basis of the degree of MR alone. Intervention remains dependent 
Mitral Regurgitation Outcomes
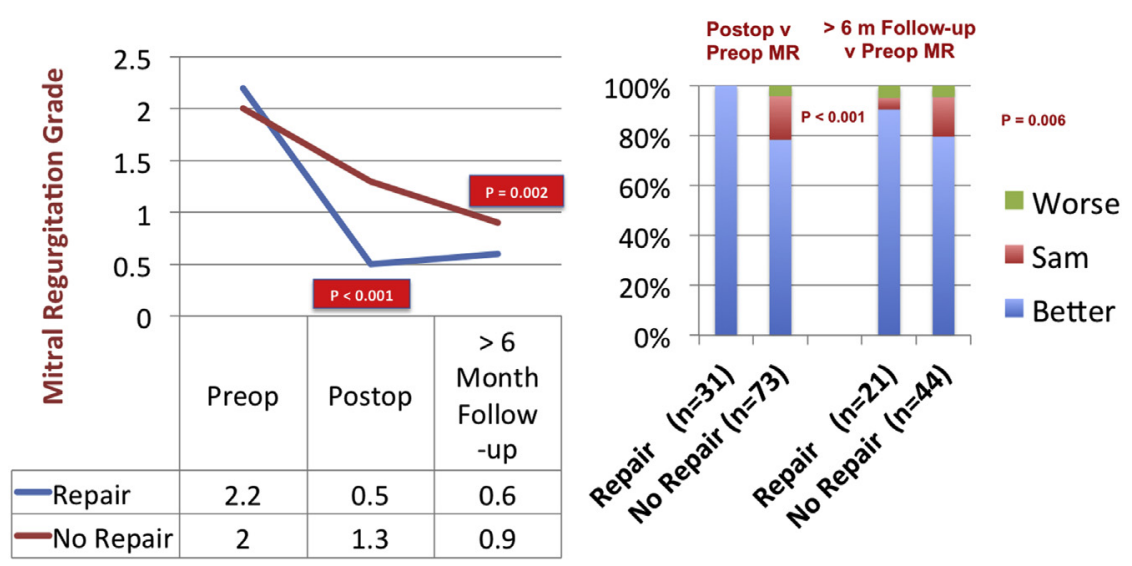

FIGURE 1. Change in mitral regurgitation $(M R)$. Preop, Preoperative; postop, postoperative.

on clinical context, patient comorbidities, left ventricle and left atrial dimensions, cause of MR, and concomitant procedures. For patients who cannot tolerate a larger operation, are undergoing concomitant $\mathrm{CABG}$, or have a dilated left ventricle or atrium, observation is an acceptable decision. It is the recommendation of this group to repair all myxomatous valves with true leaflet pathology and moderate MR in the setting of aortic root replacement. For patients with functional MR and preserved left ventricular and left atrial geometry, repair is reasonable and may yet offer further clinical benefit of reduced or absent MR demonstrated in other studies. ${ }^{7,8}$ On the whole, it is our practice to perform mitral repair for moderate MR when possible to achieve the best mitral outcomes, knowing that it does not increase surgical risk.

\section{Study Limitations}

This is a retrospective study with significant differences in the preoperative characteristics and causes of the MR between the repair and no repair groups. Long-term echocardiographic follow-up was not routinely obtained or available for all patients. This potentially creates a bias for obtaining echocardiograms in patients who had a clinical event or decompensation, but there is no clear reason why one group would be preferentially subject to this bias. MR grades were extracted from reports generally based on Doppler jets and not on more rigorous single-reviewer and vena contracta measurements. Further division of the repair and no repair groups into different causes, preoperative characteristics, and postoperative outcomes made multivariate analysis limited by small group sizes, but this is still the largest study we could find that addresses the specific but challenging question of management and outcomes of moderate MR in aortic root replacement surgery.

\section{CONCLUSIONS}

Despite increased heart failure and preoperative risk, mitral repair along with aortic root replacement had superior mitral valve outcomes, zero operative deaths, and equivalent long-term clinical outcomes compared with the no mitral repair group. This suggests that repairing moderate MR generally should be considered at the time of aortic root replacement surgery.

\section{References}

1. Unger P, Dedobbeleer C, Van Camp G, Plein D, Cosyns B, Lancellotti P. Mitral regurgitation in patients with aortic stenosis undergoing valve replacement Heart. 2010;96:9-14.

2. David TE, Armstrong S, Maganti M, Ihlberg L. Clinical outcomes of combined aortic root replacement with mitral valve surgery. J Thorac Cardiovasc Surg. 2008:136:82-7.

3. Talwar S, Mathur A, Choudhary SK, Singh R, Kumar AS. Aortic valve replacement with mitral valve repair compared with combined aortic and mitra valve replacement. Ann Thorac Surg. 2007;84:1219-25.

4. Barreiro CJ, Patel ND, Fitton TP, Williams JA, Bonde PN, Chan V, et al. Aortic valve replacement and concomitant mitral valve regurgitation in the elderly: impact on survival and functional outcome. Circulation. 2005; 112(9 Suppl):I-443-7.

5. Litmathe J, Boeken U, Kurt M, Feindt P, Gams E. Predictive risk factors in double-valve replacement (AVR and MVR) compared to isolated aortic valve replacement. Thorac Cardiovasc Surg. 2006;54:459-63.

6. Harris KM, Sundt III TM, Aeppli D, Sharma R, Barzilai B. Can late survival of patients with moderate ischemic mitral regurgitation be impacted by intervention on the valve? Ann Thorac Surg. 2002;11:1468-75.

7. Moazami N, Diodato MD, Moon MR, Lawton JS, Pasque MK, Herren RL, et al. Does functional mitral regurgitation improve with isolated aortic valve replacement? J Card Surg. 2004;19:444-8.

8. Caballero-Borrego J, Gómez-Doblas JJ, Cabrera-Bueno F, García-Pinilla JM, Melero JM, Porras C, et al. Incidence, associated factors and evolution of non-severe functional mitral regurgitation in patients with severe aortic stenosis undergoing aortic valve replacement. Eur J Cardiothorac Surg. 2008; 34:62-6.

9. Harris KM, Malenka DJ, Haney MF, Jayne JE, Hettleman B, Plehn JF, et al. Improvement in mitral regurgitation after aortic valve replacement. Am J Cardiol. 1997;80:741-5.

10. Kaczorowski DJ, MacArthur JW, Howard J, Kobrin D, Fairman A, Woo YJ Quantitative evaluation of change in coexistent mitral regurgitation after aortic valve replacement. J Thorac Cardiovasc Surg. 2013;145:341-8. 